\title{
Accuracy of assessment of gonial angle by both hemispheres of panoramic images and its comparison with lateral cephalometric radiographic measurements
}

\begin{abstract}
Background: One of the most important values in cephalometric tracing is the gonial angle which is used to measure growth pattern of patients, teeth extraction pattern in Class II patients, surgical decision in class III skeletal base patients and age estimation in forensic medicine. Gonial angle measured from panoramic radiograph is found to be more reliable than lateral cephalometric radiograph. In the latter, superimposition of the left and right sides angle, makes it difficult to measure accurately gonial angle.
\end{abstract}

Aims: I aimed at testing the similarity of left and right sides of Orthopantomography in measuring the gonial angle vs. its measurement from lateral cephalometric radiograph.

Materials and methods: This cross-sectional hospital files-based study of patients visited the Staff Clinic of College of Dentistry, Aljouf University, Sakaka; Al-Jouf, Saudi Arabia was done in the period from $1^{\text {st }}$ January 2015 to 30th September 2015. The prescribed panoramic and lateral cephalometric radiographs used and gonial angle were traced.

Results: Only measurements yielded from each of the panoramic images (right and left) vs. lateral cephalometric were statistically significant ( $\mathrm{p}<0.030$ and 0.001 , respectively).

Conclusion: The gonial angles measured from each of left and right sides of panoramic images were equally reliable but when these measurements were compared to the gonial angles measured on the cephalometric radiographs, the measurements were found statistically different.
Volume 4 Issue 4 - 2016

\author{
Ibadullah Kundi \\ Department of Orthodontist, Aljouf University College of \\ Dentistry, Aljouf University, Saudi Arabia
}

Correspondence: Ibadullah Kundi, Department of Orthodontist, Aljouf University College of Dentistry, Aljouf University, Saudi Arabia,Tel 00966 5420484I2, Fax 923000000000, Email dr.ibadullah.kundi@judent.org

Received: February 09, 2016 | Published: March 8, 2016

Keywords: gonial angle, panoramic radiography, lateral cephalometric radiography

\section{Introduction}

Professor Yrjo Paatero, in 1961, first introduced the Orthopantomography (OPG. ${ }^{1}$ It has been extensively used in dentistry for analysing the number and type of teeth present, caries, impacted teeth, root resorption, ankylosis, shape of the condyles, ${ }^{2}$ temporomandibular joints, sinuses, fractures, cysts, tumours and alveolar bone level. ${ }^{3,4}$ Panoramic radiography is advised to all patients seeking orthodontic treatment; including Class I malocclusions. ${ }^{5}$ The gonial angle is crucial in determining growth pattern of patients, ${ }^{6}$ teeth extraction pattern in Class II patients, ${ }^{7}$ decision making whether to carry out surgery in class III skeletal base patients ${ }^{8}$ and age estimation in forensic medicine. ${ }^{9}$ Recent studies have highlighted that OPG can be used to calculate the gonial angle which is a key value in cephalometric diagnosis. ${ }^{10}$ Larheim \& Svanaes ${ }^{11}$ in 1986 concluded that the gonial angle measured from a lateral cephalometric radiograph is not accurate and is difficult to measure because of right and left side superimposition. They also concluded that gonial angle measurements from panoramic radiographs are equal to those measured from dried human mandibles. ${ }^{11}$ There are two methods of constructing the gonial angle. The first is to draw a tangent on the posterior border of the ramus of the mandible and join it with another line passing through the points gonion and Gnathion. ${ }^{12}$ The second method is to draw a tangent on the lower border of the mandible and another tangent to the distal border of the ascending ramus and condyle and measure the angle in between them. ${ }^{13}$ Due to difficulty in reliably identifying gnathion on lateral cephalometric radiographs, I have used the later technique to determine the gonial angle.

The objective of this cross-sectional study was to test the reliability of measurements of the gonial angle yielded from left and right hemispheres of panoramic radiographs and compare them to the values obtained from lateral cephalograms. The rationale of performing the study is to check the possible application and reliability of panoramic radiographs for gonial angle determination by clarifying whether there is any significant difference between them and those from lateral cephalograms in an attempt to enhance the use of panoramic radiography for gonial angle measurements.

\section{Materials and methods}

Records of patients who were enrolled for treatment at the Staff Clinic of the College of Dentistry, Aljouf University, Sakaka, Al-Jouf, Saudi Arabia were included in this cross-sectional study. Each patient was given a serial number to protect his/her confidentiality. The duration of this study was 9 months, i.e., from $1^{\text {st }}$ January 2015 to 30 th September, 2015. The sample size comprised 80 patients $^{14}(50$ male 
and 30 female patients). Radiographs selected were for patients with Class I malocclusion, taken from the same apparatus. The radiographs were analysed by two experienced orthodontists. $2 \mathrm{H}$ pencil was used to draw the tangents. Panoramic and cephalometric images were acquired with a Cranex D digital X-ray unit, Version 3 (Soredex Co., Tuusula, Finland). $85 \mathrm{KVP}$ for panoramic radiograph and $73 \mathrm{KVP}$ for lateral cephalometric radiograph were used. 20 seconds exposure time was selected through $2.7 \mathrm{~mm} \mathrm{Al} \mathrm{filtration} \mathrm{for} \mathrm{both} \mathrm{types} \mathrm{of}$ radiographs. The two experienced orthodontists analysed clinically the radiographs for high quality and sharpness. Patients having history of trauma, previous facial/mandibular surgery, syndromes (affecting face/jaw) and incomplete records were excluded. Approval on the study design from the local ethical committee of the College of Dentistry, Aljouf University, Sakaka, Saudi Arabia, was obtained. The gonial angle was measured between the two tangent lines; one to the distal border of the ascending ramus and condyle and the second line to the lower border of the mandible (Figure 1). The data were analyzed by SPSS 17. Paired Student's t-test was used to compare the variables. The level of significance was set at $p \leq 0.05$. The radiographs were viewed and evaluated by two expert orthodontists. The gonial angle in the intersection of the ramal plane and mandibular plane was traced on tracing paper and measured using a protractor with 1 degree accuracy (Figure 2). Gonial angle measurement was done twice, with an interval of 1 month apart, and Dahlberg's formula was used to determine intra observer reproducibility.
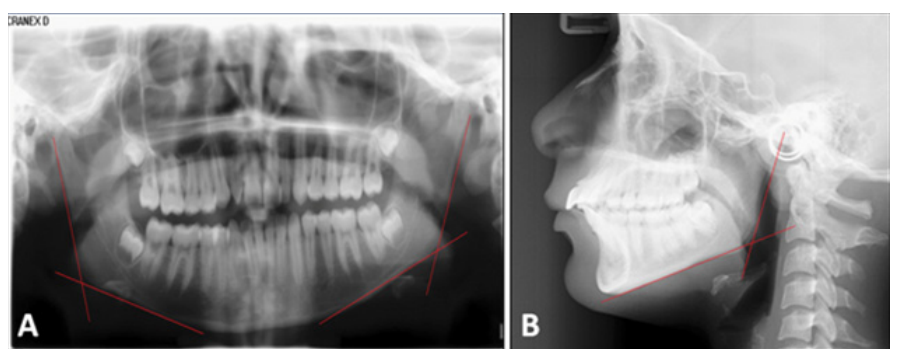

Figure I A) The approach used to measure gonial angle on a representative panoramic radiograph investigated. B) The approach used to measure gonial angle on a representative lateral cephalogram investigated.

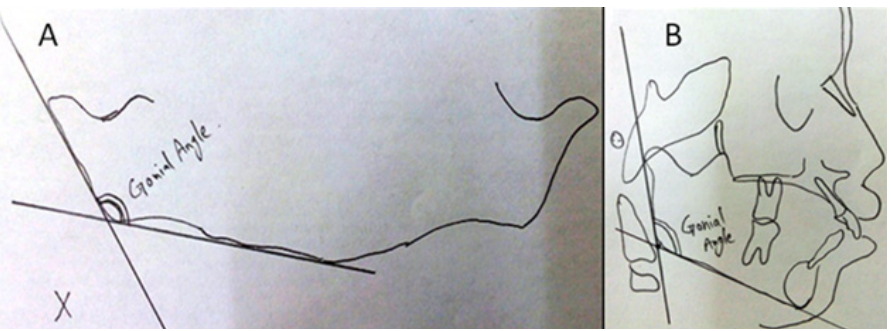

Figure 2 A) Example gonial angle tracing from a panoramic radiograph. B) Example gonial angle tracing from on a lateral cephalogram.

\section{Results}

The mean \pm standard deviations of gonial angle measured from lateral cephalometric radiographs vs. Right and left panoramic radiographs were $124.89 \pm 6.18,123.06 \pm 6.39$, and, $123.32 \pm 6.10$, respectively (Table 1). No significant statistical difference was found when right and left side Panoramic measurements were compared $(p=0.129)$. A significant difference was found when each of the panoramic measurements (right and left) was compared to lateral cephalometric measurements $(\mathrm{p}<0.035$ and 0.001 , respectively) (Table 1).
Table I Mean and standard deviations of gonial angle measured from lateral cephalometric radiographs as well as bilaterally from panoramic radiographs (right and left angles)

\begin{tabular}{llll}
\hline & Lateral Ceph & \multicolumn{1}{c}{ Panoramic right } & Panoramic left \\
\hline Mean & 124.89 & 123.06 & 123.32 \\
SD & 6.18 & 6.39 & 6.10 \\
\hline
\end{tabular}

SD: Standard deviation

Table 2 Comparison of OPG and Lateral Ceph. *= Significant difference, paired Student's " $t$ " test at $\mathrm{P} \leq 0.05$

\begin{tabular}{lllll}
\hline & Mean & ISD & P value \\
\hline Pair I Lateral Ceph - Panoramic Right & 2.212 & 4.277 & $0.035 *$ \\
Pair 2 Lateral Ceph - Panoramic Left & 1.600 & 3.723 & $0.001 *$ \\
Pair 3 Panoramic Right - Panoramic Left & 0.612 & 4.510 & 0.129 \\
\hline
\end{tabular}

\section{Discussion}

This study was undertaken to test the reliability of measurements of the gonial angle yielded from left and right hemispheres of panoramic radiographs as compared to the values obtained from lateral cephalograms in patients with ages ranging between 15-25 years having class I malocclusion. The gonial angle depicts the form and shape of the mandible, has a pivotal role in forecasting future mandibular growth and it also has certain effects on profile, changes, growth and the position of the mandibular anterior teeth. ${ }^{15}$ The basic reason of conducting the current study was to enhance the application of panoramic radiography in clinical practice to determine the gonial angle. A few previous studies done to investigate the same aim found no significant difference between gonial angle values measured from panoramic radiographs and those yielded from lateral cephalometric images. ${ }^{16}$ However, the current study revealed different results. This study is in agreement with a previous study done by Bhullar in 2014, which demonstrated equal values for measurements from both sides of panoramic radiographs. ${ }^{16}$

Clinical usefulness of panoramic radiography for determining the gonial angle is confirmed by previous studies. This is due to the facts that the left and right side gonial angles are not superimposed on panoramic radiographs, unlike lateral cephalograms which exhibit superimposition. ${ }^{10,17-19}$ Mattila et al..$^{15}$ in an old study in 1971 took measurements of gonial angle on cephalograms, panoramic radiographs and dried skulls. They showed that the measurements for right and left gonial angles from panoramic images were equal to the angles measured on dry skulls. They further reported that the means of the measurements made on cephalograms showed lower accuracy than those of the panoramic radiographs when compared to the dry mandibles' measurements. Nohadani et al. ${ }^{20}$ in 2008 compared vertical facial and dentoalveolar changes as measured on panoramic and lateral cephalometric radiographs. They stated that panoramic radiographs are not useful for measuring the vertical facial dimension changes. And, angular values from panoramic radiograph are more reliable than vertical values. This was because in the posterior and lateral aspects of the mandible, the angular values are not influenced by image distortion inherent with panoramic radiography. In a most recent study, Araki et al. ${ }^{21}$ in 2015 compared gonial angles measured from 49 panoramic radiographs with the gonial angle on lateral cephalometric radiographs taken for 2 dry mandibles. The gonial angle measurements were slightly smaller on the panoramic radiographs 
than on the lateral cephalometric radiographs. The mean gonial angle was $115.1 \pm 5.2^{\circ} \mathrm{C}$ on the panoramic radiographs and $122.2 \pm 6.4^{\circ} \mathrm{C}$ on lateral cephalometric radiographs.

\section{Conclusion}

No statistically significant difference was found when right and left side panoramic gonial angle measurements were compared. On the other hand, panoramic measurements (right and left) and lateral cephalometric measurements were not equal.

\section{Limitations of the study}

I. Since sample size was small, the reliability and strength of the study for generalization requires a large sample size.

II. Gender distribution was not equal (50 male and 30 female).

III. Limitations related to lateral cephalometric images are related to the errors in identification of landmarks which could result in errors during tracing. These factors could decrease the reliability of cephalometric.

\section{Funding}

None.

\section{Acknowledgements}

None.

\section{Conflicts of interest}

Author declares that there is no conflict of interest.

\section{References}

1. White SC, Pharaoh MJ. Oral radiology: principles and interpretation. $4^{\text {th }}$ ed. St. Louis, USA: Mosby; 2000. p. 205-206.

2. Staley RN, Ruske NT. Essentials of orthodontics: diagnosis and treatment $1^{\text {st }}$ ed. A John Wiley \& Sons Inc., West Sussex; 2011. p. 57-61.

3. Bishara SE. Textbook of orthodontics. ( $2^{\text {nd }}$ edn), W.B. Saunders, Philadelphia, USA; 2007. p. 112.

4. Graber LW, Vanarsdall RL, Vig KWL. Current principles and techniques. $5^{\text {th }}$ ed. St. Louis, USA: Mosby; 2012. p. 112-113.

5. Cobourne MT, Flemming PS, DiBiase AT. Clinical cases in orthodontics. $1^{\text {st }}$ ed. John Wiley \& Sons Inc., West Sussex; 2012. p. 16-17.

6. Xiao D, Gao H, Ren Y. Craniofacial morphological characteristics of Chinese adults with normal occlusion and different skeletal divergence. Eur J Orthod. 2011;33(2):198-204.
7. Nanda SK. Growth patterns in subjects with long and short faces. Am J Orthod Dentofacial Orthop. 1990;98(3):247-258.

8. Tahmina K, Tanaka E, Tanne K. Craniofacial morphology in orthodontically treated patients of class III malocclusion with stable and unstable treatment outcomes. Am J Orthod Dentofacial Orthop. 2000;117(6):681-690.

9. Upadhyay RB, Upadhyay J, Agrawal P, et al. Analysis of gonial angle in relation to age, gender, and dentition status by radiological and anthropometric methods. J Forensic Dent Sci. 2012;4(1):29-33.

10. Shahabi M, Ramazanzadeh BA, Mokhber N. Comparison between the external gonial angle in panoramic radiographs and lateral cephalograms of adult patients with class 1 malocclusion. J Oral sci. 2009;51(3):425429.

11. Larheim TA, Svanaes DB. Reproducibility of rotational panoramic radiography: mandibular linear dimensions and angles. Am J Orthod Dentofacial Orthop. 1986;90(1):45-51.

12. Koeppe K. Forecast mandibular development using the ortho pantomogram. Fort shear of orthodontia. 1973;34:316-322.

13. Dahan J, Jesdinsky HJ. The valuation of ortho pantomogram for kephalometrischeuntersuchungen in orthodontia. Stoma. 1968;21: 126138, 200-206.

14. Zangouei-Booshehri M, Aghili HA, Abasi M, et al. Agreement between panoramic and lateral cephalometric radiographs for measuring the gonial angle. Iran J Radiol. 2012;9(4):178-182.

15. Mattila K, Altonen M, Haavikko K. Determination of the gonial angle from the orthopantogram. Angle orthod. 1977;47(2):107-110.

16. Bhullar MK, Uppal AS, Kochhar GK, et al. Comparison of gonial angle determination from cephalograms and orthopantomogram. Indian J Dent. 2014;5(3):123-126.

17. Alhaija ES. Panoramic radiographs: determination of mandibular steepness. J Clin Pediatr Dent. 2005;29(2):165-166.

18. Raustia AM, Salonen MA. Gonial angles and condylar and ramus height of the mandible in complete denture wearers-a panoramic radiograph study. J Oral Rehabil. 1997;24(7):512-516.

19. Ceylan G, Yanikoglu N, Yilmaz AB,et al. Changes in the mandibular angle in the dentulous and edentulous states. J Prosthet Dent. 1998;80(6):680684 .

20. Nohadani N, Ruf S. Assessment of vertical facial and dentoalveolar changes using panoramic radiography. Eur J Ortho. 2008;30(3):262-268.

21. Araki M, Kiyosaki T, Sato M, et al. Comparative analysis of the gonial angle on lateral cephalometric radiographs and panoramic radiographs. $J$ Oral Science. 2015;57(4):373-378. 